\title{
The Rebound Effect and Energy Efficiency Policy
}

Kenneth Gillingham, David Rapson, and Gernot Wagner

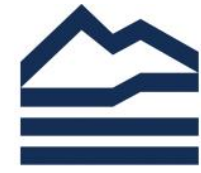




\title{
The Rebound Effect and Energy Efficiency Policy
}

\author{
Kenneth Gillingham, David Rapson, and Gernot Wagner
}

\begin{abstract}
What do we know about the size of the rebound effect? Should we believe claims that energy efficiency improvements lead to an increase in energy use? This paper clarifies what the rebound effect is, and provides a guide for economists and policymakers interested in its magnitude. We describe how some papers in the literature consider the rebound effect from a costless exogenous increase in energy efficiency, while others examine the effects of a particular energy efficiency policy - a distinction that leads to very different welfare and policy implications. We present the most reliable evidence available quantifying the energy efficiency rebound, and discuss areas where estimation is extraordinarily difficult. Along these lines, we offer a new way of thinking about the macroeconomic rebound effect. Overall, the existing research provides little support for the so-called "backfire" hypothesis. Still, much remains to be understood, particularly relating to induced innovation and productivity growth.
\end{abstract}

Key Words: energy efficiency, rebound effect, take-back effect, backfire, Jevons Paradox JEL Classification Numbers: H23, Q38, Q41

(C) 2014 Resources for the Future. All rights reserved. No portion of this paper may be reproduced without permission of the authors.

Discussion papers are research materials circulated by their authors for purposes of information and discussion. They have not necessarily undergone formal peer review. 


\section{Contents}

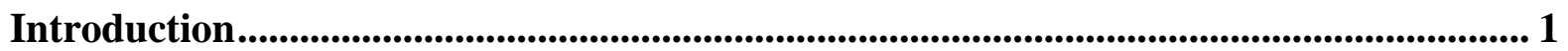

Defining the Rebound Effect................................................................................................................ 2

Channels of the Rebound Effect: Microeconomic ........................................................................... 5

Substitution and Income Effects ................................................................................ 5

Measuring Microeconomic Rebound Effects ……………........................................... 8

Channels of the Rebound Effect: Macroeconomic ................................................................. 14

Defining Macroeconomic Rebound Effects............................................................... 15

Quantifying Macroeconomic Rebound Effects ......................................................... 17

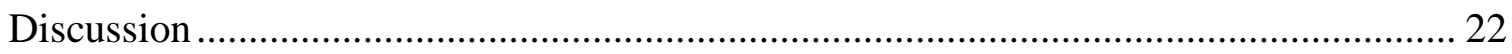

Conclusions and Lessons for Policy .............................................................................................. 23

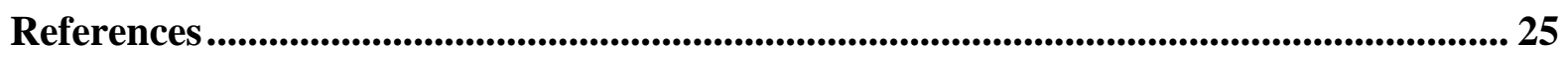




\title{
The Rebound Effect and Energy Efficiency Policy
}

\author{
Kenneth Gillingham, David Rapson, and Gernot Wagner*
}

\section{Introduction}

Buy a more fuel-efficient car, drive more. That is the most well-known intuition for the rebound effect. Its existence is clear, and it has been for a long time: Jevons (1865) hypothesized that greater energy efficiency may even lead to a "backfire" of increased industrial energy use. However, the magnitude of the rebound effect is much less clear, and estimates show incredible variation — with stark implications for energy efficiency policy.

The differences in estimates of the rebound effect stem from its varying definitions, as well as variation in the quality of data and empirical methodologies used to estimate it. The goal of this paper is to clarify the definition of each of the channels of the rebound effect and critically assess the state of the literature that estimates its magnitude. We emphasize the difference between the rebound effect from a costless exogenous energy efficiency improvement - what we call a 'Zero-Cost Breakthrough' (ZCB) — and the rebound effect from an actual energy efficiency policy - a 'Policy-Induced Improvement' (PII). Acknowledging this distinction can help with interpreting estimates in the literature. The common approach of using empirically estimated fuel price or operating cost elasticities of demand as one measure of the rebound effect should be treated with caution. However, since there is a reliable literature estimating these elasticities, they often provide the most useful information available.

When we consider the total rebound effect, and especially effects that may occur at the macroeconomic level, reliable empirical estimates are much harder to come by. We review estimates in the literature, where available, and provide a conceptual discussion to help

\footnotetext{
*Yale University; e-mail: kenneth.gillingham@yale.edu; University of California, Davis; e-mail: dsrapson@ucdavis.edu; Environmental Defense Fund, adjunct associate professor at Columbia University's School of International and Public Affairs, and research associate at Harvard Kennedy School; e-mail: gwagner@edf.org. Acknowledgments: The research for this paper arose from an Environmental Defense Fund workshop. Our special thanks go to Matthew Kotchen, who helped conceptualize this project. We thank Blake Alcott, Severin Borenstein, Dallas Burtraw, Nathan Chan, Frank Convery, Manuel Frondel, Nathaniel O. Keohane, Reid Leifset, Derek Lemoine, Kenneth Small, Thomas Sterner, Karen Turner, Colin Vance, Matthew Zaragoza-Watkins, Richard J. Zeckhauser, the editor, and two anonymous referees for comments, as well as Jonathan Camuzeaux, Ruiwen Lee, and Paige Weber for excellent research assistance. All remaining errors are our own. The authors are not aware of any conflicts of interest. Kenneth Gillingham was formerly a research assistant at Resources for the Future.
} 
contextualize the existing estimates and provide intuition into magnitudes where empirical evidence is scarce. Our review of the literature leads us to conclude that a continued focus within policy debates on backfire is largely unwarranted, and distracts from the issue of utmost importance: evaluating the economic efficiency of energy efficiency policies. The rebound effect is just one component of this more important analysis. Moreover, the rebound effect usually improves economic efficiency, so policies aimed at mitigating the rebound effect, as have been discussed in the literature (e.g., van den Bergh (2011)) and policy community (e.g., Gloger (2011)), are likely counterproductive from a welfare perspective. However, in assessing the potential for backfire, path dependency in innovation is a 'wild card.' Despite decades of research on the rebound effect, there is still much to be learned, and we point to several highpotential avenues.

The paper is structured as follows. We first define the different components of the rebound effect. We then briefly summarize quantitative evidence on the different channels of rebound effect, and discuss challenges to identifying causal rebound effects in each. Finally, we conclude with implications for energy efficiency policy. Along the way, we point out popular misconceptions of about the rebound and how to address them.

\section{Defining the Rebound Effect}

The classic way to think about the rebound effect conjectures an improvement in energy efficiency and compares the achieved reductions in energy use to the forecasted reductions in energy use that ignore consumer and market responses. Such consumer and market-wide responses are likely to occur because the energy efficiency improvement changes relative prices (and real income). The rebound effect is expressed as a percentage of the forecasted reduction in energy use that is 'lost' due to the sum of consumer and market responses.

To illustrate, consider an air conditioner with annual electricity use of $100 \mathrm{kWh} /$ year. Suppose a more efficient air conditioner shaved $10 \mathrm{kWh} /$ year off this total before accounting for any consumer and market responses. If these responses increased electricity use by $1 \mathrm{kWh} / \mathrm{year}$, 
then the rebound effect would be equal to 10 percent, since 1 of the $10 \mathrm{kWh}$ per year in expected energy savings would be retaken by consumer and market responses. ${ }^{1}$

This broad definition captures the essence of the rebound effect, but abstracts from how energy efficiency is improved and what happens to other product attributes. The literature handles these issues remarkably differently, which can lead to confusion around what the rebound effect is exactly, how to estimate it, and how to interpret the results. A first-order distinction is whether we are considering (1) an exogenous increase in energy efficiency holding other attributes constant or (2) a change in energy efficiency that is bundled with changes in other product attributes. The latter may induce a change in the energy service provided and perhaps also a change in the cost of the product. ${ }^{2}$

To illustrate this distinction, first consider a ZCB: a scenario where an innovation allows a product (e.g., appliance) manufacturer to increase energy efficiency costlessly, while holding all other attributes of the product the same. The subsequent consumer and market responses are a pure conception of the rebound effect, for they capture only responses induced by an improvement in energy efficiency.

In contrast, consider a PII: a scenario where an energy efficiency policy requires manufacturers to improve the energy efficiency of a particular product. In this case, the energy efficiency improvement may be costly, potentially raising the price of the product. Concurrently, it may induce or even necessitate changes in other attributes of the product, such as size, weight or capacity. In this case both the price and the energy service provided by the product may change along with energy efficiency.

There may be a continuum between ZCB and PII, whereby a rebound effect captures some, but not all, of the changes from a policy. Such intermediates may be more difficult to interpret for providing policy insights, depending on the specific circumstances. Accordingly, we focus our discussion on the two extremes: ZCB and PII.

\footnotetext{
${ }^{1}$ Here we follow the literature by defining the rebound effect with respect to energy. One could analogously define the rebound effect with respect to emissions (Thomas et al. 2013), which in many cases is proportional to the energy rebound. Exceptions include biofuels policies that lead to indirect land use emissions, or policies that lead to fuel switching, for example from coal to natural gas, and, thus, from carbon to methane emissions.

2 Energy is a derived demand from the consumers' demand for energy services (e.g., miles driven in a particular car, refrigeration, etc). These energy services themselves may change along with the attributes of a product (e.g., a refrigerator with an ice maker provides a different energy service than one without).
} 
For both estimation and policy purposes, it is crucial to distinguish between ZCB and PII. Suppose we want to estimate a response attributed directly to an energy efficiency improvement. Then the ZCB scenario is likely to be a better guide to the effect. Any empirical estimation that controls for all of the key attributes of a product is aiming to identify this effect. This approach is the most common way to estimate what most authors call the rebound effect. In contrast, if we are interested in the overall effect of a policy - the bundle of changes that occurs, including but not limited to energy efficiency - then PII is the appropriate approach. The goal would be to estimate a compound effect that combines the energy savings from the efficiency improvement with the energy adjustments due to the change in attributes and cost of the product. It may even capture changes in sales of the product or other consequences, depending on the empirical setting. To calculate the PII rebound effect, one could examine the difference in the forecasted energy savings based on a simple engineering calculation and the empirically estimated effect. This result, while more appropriate for considering the energy implications of specific policies, is not generally equivalent to the perhaps more 'pure' conception of the rebound effect.

Which of these two approaches should we prefer for policy analysis? The answer depends. Isolating the effect of an exogenous energy efficiency improvement on the consumer and market responses (ZCB) provides clear guidance for policymakers on how changes in energy efficiency alone would change energy use. The results are likely to be more applicable across settings by virtue of holding constant potentially confounding variables. They may be used to demonstrate the degree to which the rebound effect improves social welfare by providing energy services that consumers value. Moreover, if policy-induced energy efficiency improvements are associated with only negligible costs and changes in attributes, estimates for ZCB may be similar to those for PII.

However, in most cases when there is an energy efficiency policy there are also changes in costs and attributes, the responses to which are difficult to disentangle empirically. To analyze such an energy efficiency policy it is essential to know all of the pertinent consumer and market responses to the improved efficiency, changed attributes, and increased cost. All these different responses comprising the policy's effect play a role in what ultimately matters most: the social welfare effects of the policy.

The distinction between the two approaches for thinking about the rebound effect provides important context for our review of the literature. Most studies that aim to estimate the rebound effect have an exogenous increase in energy efficiency in mind; fewer are examining an actual energy efficiency policy. 


\section{Channels of the Rebound Effect: Microeconomic}

When energy efficiency improves, the price of usage changes, so both substitution and income effects influence consumers' consumption and corresponding energy use. However, retrieving measurements of these effects is far from trivial. We review the theoretical microeconomic foundation for the rebound effect and show how this is translated into empirical estimates. The sheer number of estimates of rebound effects in the literature is impressive and the empirical approaches diverse. We filter them using modern empirical standards. Not all 'old' estimates lack credibility, and not all 'new' estimates should be believed. However, the changing standards motivate our decision not to perform an exhaustive review of this expansive literature, but instead focus on what we consider to be most reliable estimates. Our focus here is on consumers; we will discuss production as part of the macroeconomic rebound discussion.

\section{Substitution and Income Effects}

In the context of a costless energy efficiency improvement, the decline in the cost of the energy services implies that consumers will make a series of four adjustments to their consumption bundle. ${ }^{3}$ These four adjustments may in turn affect their derived demand for energy. First, consumers will substitute towards the more energy-efficient product, which is now relatively less expensive. Second, consumers will substitute away from other relatively expensive goods (and more broadly change their bundle of consumption towards complements and away from substitutes of the energy efficient product). Third, the lower effective price for the energy service increases the consumer's purchasing power, so they will further increase consumption of the more energy-efficient product (assuming it is a normal good). Finally, the increased purchasing power means that consumers will increase consumption of other normal goods as well. These effects each serve to either increase or decrease the amount of energy used for the consumption bundle.

\footnotetext{
${ }^{3}$ Readers interested in an expanded discussion and slightly more technical treatment should refer to Borenstein (2015).
} 


\section{Answers to Popular Misconceptions about the Rebound Effect}

\section{No Causality, No Rebound}

Energy demand can increase for a whole host of reasons, with economic growth and improved energy technology chief among them. But correlation is not causation, and causation must be established before anything can be labeled "rebound."

\section{Developing Countries' Rebound Could be Either Larger or Smaller than in Developed Countries}

We might expect total energy demand to increase more quickly in developing countries, but the relative size of the rebound effect is theoretically ambiguous. It depends on the link between energy services and other goods, like food and clothing. If they are substitutes, we could see a high rebound. If they are complements - and plausible stories point in that direction — rebound would be lower. Ultimately, the truth is an empirical matter. Thus far, most estimates from developing countries are in line with those from developed countries.

\section{Total Rebound Effect is Likely Smaller than the Sum of Its Parts}

Rebound consists of four components: direct, indirect, macro price, and macro growth. A full assessment needs to consider all four causal pathways. Yet the total effect may be smaller than their sum, for estimates may include multiple channels. In some situations, different channels may subsume or offset each other.

\section{Don't Mitigate Rebound, Maximize Welfare}

Perhaps the largest misconception is one that focuses on the perceived 'evil' of the rebound itself. This notion implicitly has in mind energy-minimization as the objective, rather than welfare-maximization. Rebound occurs from consumers responding to changes in relative prices, so by revealed preference, it provides welfare benefits (relative to no response). In an energy efficiency policy evaluation, including the rebound effect is likely to add to the tally of benefits and will only increase costs due to external costs from the additional usage.

These effects do not map perfectly into the terms most commonly used in the literature. The 'direct rebound effect' is generally defined as the change in energy use resulting from the combined substitution and income effects on the demand for energy-efficient product. This definition is convenient because economists typically estimate elasticities of demand, such as the elasticity of demand for air conditioning with respect to the operating cost of the air conditioner. An operating cost elasticity of demand tells us how much additional air conditioning consumers will use if their operating cost changes on the margin, while holding all other attributes constant 
(ZCB approach). For example, if the elasticity of demand with respect to the operating cost is 0.5 , then 50 percent of the reduction in energy use from an improvement in energy efficiency on the margin will be taken back by the substitution and income effects increasing the usage (ignoring the substitution and income effects on other goods). Importantly, the direct rebound effect ignores any changes to the demand for other goods due to either the change in relative prices or purchasing power. Nonetheless, the direct rebound is useful for understanding the consumer response to an energy efficiency increase.

The literature examines demand for all other goods via the 'indirect rebound effect.' However, the literature is inconsistent in the usage of this term. Some studies include any changes in energy use from changes in demand for other goods, including from the substitution and income effects, as well as any embodied energy used to create the energy efficiency improvement (Azevedo 2014). Other studies are even broader and include any additional energy use not captured by the direct rebound (including macroeconomic effects) as part of the indirect rebound (Sorrell et al. 2008). However, it is most common to refer to the indirect rebound effect as only the income effects on the consumption of all other goods. For example, buyers of a more fuel-efficient vehicle may decide to spend the savings on an extra flight for vacation - another energy-intensive activity. Or they may spend the savings on something much less energy intensive, such as books and movies. The sign and magnitude of the effect is determined by the difference in the energy intensity (per dollar) between the energy-efficient product (prior to the efficiency improvement) and other goods consumed on the margin. Importantly, this more common definition of the indirect rebound ignores the substitution effects on other goods from the decrease in the price of using the more energy-efficient product. These are typically implicitly assumed away as being insignificant. Similarly, it is also common to ignore any cost of the efficiency improvement, even though such a cost would change the income effects, reducing (increasing) them if the energy-efficient product before the improvement is more (less) energy intensive than the marginal consumption bundle (Borenstein 2015).

Overall, the adjustments described above are no different from any other adjustments that consumers make when confronted with a change in relative prices. So, by revealed preference, consumers are enjoying private surplus gains. It follows that a net welfare decrease from a rebound effect would only be possible if the external costs associated with these adjustments 
outweigh the private gains. This is possible, but requires externalities that are large enough to outweigh the increased consumer surplus from the substitution and income effect responses. ${ }^{4}$

\section{Measuring Microeconomic Rebound Effects}

As the microeconomic rebound is made up of substitution and income effects across all goods, a full attempt at measurement would require estimating these effects for all goods in the economy - a daunting task. Instead, most studies focus on estimating the price elasticity of demand for the more energy-efficient product (ZCB), ignoring the demand for other goods. A few studies estimate the effect of a policy (PII), although again generally ignore effects on other goods throughout the economy. There are also a few estimates of the income effects from changing the energy consumption of all other goods, generally based on the average consumption bundle, rather than the marginal consumption bundle. We are not aware of any studies that estimate these effects jointly using comparable data sources. This may be problematic since under common assumptions a greater increase in demand for the energy efficient product (i.e., direct rebound) implies a smaller increase in demand for other goods (i.e., substitution and income effects on other goods) (Chan et al. 2014).

Before moving to estimates, several further words of caution are in order. First, to provide reliable guidance, it is critical that studies estimate a causal effect. This is particularly relevant to the use of demand elasticities to quantify the rebound effect. Many studies estimating demand elasticities do not hold up to modern standards of identification, and fail to address standard empirical issues such as simultaneity and other endogeneity concerns. For instance, studies that rely on cross-sectional variation in fuel prices or operating costs may have difficulty controlling for unobserved heterogeneity in a cross-section. Such studies, even if well-executed, tend to find demand to be much more elastic than studies not relying on cross-sections (e.g., see West (2004)).

Second, elasticities of demand estimated using variation in fuel prices may not necessarily provide insight into the response to changes in energy efficiency. Under standard neoclassical assumptions, the utilization of an energy-using good is based on the operating cost (i.e., the fuel price divided by the energy efficiency), so both a change in the fuel price and a change in the energy efficiency of the good would change the operating cost in identical

\footnotetext{
${ }^{4}$ See Chan and Gillingham (2014) for a more thorough investigation of these welfare effects.
} 
(opposite) ways. Thus, it is common to describe the fuel price elasticity of demand as the direct rebound effect. However, in settings with multiple energy services using the same fuel, this equality does not hold (Chan et al. 2014). Furthermore, recent evidence suggests that in the context of passenger transportation, consumers may respond comparatively less to changes in energy efficiency than to changes in fuel price (Gillingham 2011). This may occur because fuel prices are more salient, since consumers see them every time they pay their energy bill. In this case, using the fuel price elasticity of demand would overestimate the direct rebound effect. We view this as an open area of research, for other studies show either no asymmetry in response (Frondel et al. 2013) or an even greater response to changes in energy efficiency than fuel price (Linn 2013). One reason we might see a greater response to changes in energy efficiency is the perceived longevity of such changes. Li et al. (2014) finds that gasoline taxes appear to be more salient than fuel prices, perhaps due to perceived longevity.

Third, the consumer response to any change in usage costs may vary based on the timeframe of the response. When fuel prices change, in the short run consumers can choose how many trips to take, what route to take, which vehicle to take if they have multiple vehicles, and whether to take public transportation if it is available. In the medium run, they can purchase or scrap vehicles, and in the long run they can choose where to live and work. For electricity consumption when electricity prices change, consumers can immediately decide how much to cool the air with air conditioning. In the medium run they can choose how many air conditioners to own and which rooms to put them in. In the longer run, they could move to a different home altogether. In both cases, long-run energy demand is likely more elastic than short-run demand.

Finally, each study of price elasticities is from a particular time and place. Demand could vary depending on the conditions in the setting of interest. For example, Gillingham (2014) shows that the elasticity of driving demand with respect to the price of gasoline exhibits noticeable heterogeneity across different counties in California. One could imagine that there would be even greater differences when examining a developing country or a country with an extensive public transportation system. The bottom line is that even if an elasticity estimate is internally valid, we should be cautious applying it elsewhere without considering its external validity. 


\section{Elasticities in Developed Countries}

Given the above caveats, what is the state of the literature on relevant elasticities that can guide economists and policymakers? Table 1 lists a selection of recent reliable estimates of elasticities that we believe provide guidance in developed countries. ${ }^{5}$ Our review focuses on overall demand or household-level demand due to the paucity of studies on commercial and industrial demand.

The studies in Table 1 were selected both because they are more recent and use rigorous empirical methods such as panel data methods, experimental designs, and quasi-experimental approaches. They make at least a reasonable attempt to address potential endogeneity concerns or convince the reader of internal validity. They tend not to rely on cross-sectional variation. All provide either short-run or medium-run estimates. As is pointed out in Hamilton (2009) and Gillingham (2011), including a lagged dependent variable to distinguish between short-run and long-run responses relies on strong assumptions. Yet, nearly all estimates of long-run responses are based on an OLS regression with a lagged dependent variable or are based on cross-sectional variation (with the assumption that it is capturing a long-run equilibrium). Thus, we feel most comfortable with the reliability of the short-run and medium-run estimates.

The primary theme that emerges from these studies is that the short-run and medium-run elasticities of demand for gasoline/driving and electricity are generally in the range of - 0.05 to 0.4. Ignoring the caveats above, these would suggest a direct rebound effect on the order of 5 to 40 percent, with most of the studies falling in the range of 5 to 25 percent. These studies all focus on gasoline or electricity use and it may not be appropriate to apply the estimates to other energy services, including those that use other fuels, such as natural gas or heating oil. For example, there may also be a direct rebound effect for space and water heating. Unfortunately, the evidence on the price-elasticity of demand for other energy services is remarkably scant, with all of the published papers we could find over a decade old and using limited data. The reviews of the older literature by Sorrell (2007) show wide ranges for most residential energy services. In our view, new studies are needed on these other energy services. Just as importantly, new studies are needed to help us understand how large the error might be from using own-price elasticities for the direct rebound.

\footnotetext{
${ }^{5}$ For more inclusive reviews of estimates of elasticities in different sectors, see Greening et al. (2000); Sorrell (2007); Jenkins et al. (2011); and Gillingham (2011). Not surprisingly, these reviews show large ranges of estimates in most sectors.
} 
Table 1. Selected More Reliable Elasticity Estimates from Developed Countries

\begin{tabular}{|c|c|c|}
\hline Study & Type of price elasticity & $\begin{array}{l}\text { Estimated } \\
\text { Value }\end{array}$ \\
\hline Allcott (2011) & $\begin{array}{l}\text { Illinois short-run elasticity of electricity demand } 2003 \text { \& } \\
2004\end{array}$ & -0.1 \\
\hline Barla et al. (2009) & Canada short-run elasticity of VMT demand 1990-2004 & -0.08 \\
\hline Frondel et al. (2013) & Germany short-run elasticity of VMT demand 1997-2009 & $-0.458^{\ddagger}$ \\
\hline Gillingham (2014) & $\begin{array}{l}\text { California medium-run new vehicle elasticity of VMT } \\
\text { demand 2001-2009 }\end{array}$ & -0.23 \\
\hline Hughes et al. (2008) & US short-run elasticity of gasoline demand 1975-1980 & -0.21 to -0.34 \\
\hline Hughes et al. (2008) & US short-run elasticity of gasoline demand 2001-2006 & $\begin{array}{l}-0.034 \text { to } \\
-0.077\end{array}$ \\
\hline Ito $(2014)$ & $\begin{array}{l}\text { California medium-run elasticity of electricity demand } \\
\text { 1999-2007 }\end{array}$ & -0.088 \\
\hline Jessoe et al. (2013) & $\begin{array}{l}\text { Connecticut short-run elasticity of electricity demand } \\
2011\end{array}$ & -0.12 \\
\hline Small et al. (2007) & US short-run elasticity of VMT demand 1966-2001 & $-0.045^{\gamma}$ \\
\hline \multicolumn{3}{|c|}{$\begin{array}{l}\text { Notes: All electricity demand elasticity estimates are for residential customers. } \\
\gamma \text { We use the estimate from the latest period; earlier elasticities were higher in absolute value. } \\
{ }^{\ddagger} \text { We report the fixed effects estimate, which we deem most reliable. }\end{array}$} \\
\hline
\end{tabular}

It also may be inappropriate to apply the estimates in Table 1 to other regions. Most of the studies are in the United States, which has different circumstances than many other countries, both developed and developing. This is illustrated by the results in Frondel et al. (2013), which uses data from Germany, a country with better public transportation and higher gasoline prices than the United States. Perhaps not surprisingly, the results in Frondel and Vance show a more elastic response in driving to gasoline price changes than the other studies in Table 1.

\section{Elasticities in Developing Countries}

When we move beyond the developed world, the results in Table 1 may be less directly applicable. In developing countries one might hypothesize greater responsiveness, and thus a greater direct rebound effect, due to greater unmet demand for energy services. However, there are a variety of country-specific factors that affect responsiveness in any given market, such as the wealth of those who own vehicles or appliances.

In our review, we found a surprising number of papers estimating elasticities of usage for durable goods in low and middle income countries. Not surprisingly, the authors writing these papers often face severe data limitations and measurement error in the data. These studies also 
rarely meet modern standards for identification in applied economics and the caveats described above certainly apply.

In Table 2, we present a representative selection of the studies published in peer-reviewed journals. These are not filtered for reliability as in Table 1, since nearly all face data limitations and should probably not be viewed as causal estimates.

As can be seen in Table 2, the estimates of demand elasticities in developing countries range widely, with the most common range on the order of -0.1 to -0.4 in the short-run. Despite the limitations in these studies, we were interested to find that the estimated elasticities are in the same range as the developed countries' estimates.

\section{Estimated Policy-Induced Improvements}

Using the price elasticity of demand as a measure of the substitution and income effects for the good receiving the energy efficiency improvement is less helpful for understanding the net effect of all of the changes that could occur with a policy-induced energy efficiency improvement. Fortunately, there is some recent literature we can draw upon.

Davis (2008) analyzes a field experiment in which households are given more efficient clothes washers and finds a price elasticity of clothes washing of -0.06 . This estimate is similar to, but not quite the same as a ZCB because the brand new clothes washers the households were given were larger and gentler on clothes. In fact, the increase in utilization of the clothes washers (i.e., more clothes being washed overall) came about from households running more clothes in a wash. This estimate is, however, capturing the direct rebound effect of a PII from an energy efficiency policy that provides free energy efficient clothes washers. It captures all of the effects from both the change in energy efficiency and change in energy (i.e., clothes washing) service.

Davis et al. (2015) similarly examines the net effect of all of the changes that occur along with an energy efficiency policy. Specifically, Davis et al. examines a program in Mexico that provides direct cash payments and subsidized financing to consumers replacing old air conditioners and refrigerators with new energy efficient appliances, much like the cash-forclunkers program for vehicles in the United States. The results indicate a very large response from the replacement of old appliances with new energy-efficient ones: electricity use drops by only seven percent after refrigerator replacement and actually increases after replacement of the air conditioner. In this setting, there is potentially a very large change in the energy service, as well as an income effect from the transfer, both conspiring to lead to a large rebound effect from this PII. 


\section{Table 2. A Representative Sample of Recent Price Elasticity Estimates from Low and Middle Income Countries}

\begin{tabular}{|c|c|c|}
\hline Study & Type of elasticity & Estimated Value \\
\hline Al-Faris (2002) & $\begin{array}{l}\text { Gulf Cooperation Council short-run elasticity of total } \\
\text { electricity demand 1970-1997 }\end{array}$ & -0.09 \\
\hline Alves et al. (2003) & $\begin{array}{l}\text { Brazil short-run elasticity of gasoline demand 1974- } \\
1999\end{array}$ & -0.09 \\
\hline Atakhanova et al. (2007) & $\begin{array}{l}\text { Kazakhstan short-run elasticity of electricity demand } \\
\text { 1994-2003 }\end{array}$ & $-0.128^{\ddagger}$ \\
\hline Athukorala et al. (2010) & $\begin{array}{l}\text { Sri Lanka short-run elasticity of total elasticity demand } \\
1960-2007\end{array}$ & -0.16 \\
\hline Ben Sita et al. (2012) & $\begin{array}{l}\text { Lebanon short-run elasticity of gasoline demand 2000- } \\
2010\end{array}$ & -0.623 \\
\hline Crotte et al. (2010) & $\begin{array}{l}\text { Mexico short-run elasticity of gasoline demand 1980- } \\
2006\end{array}$ & 0 to -0.15 \\
\hline Halicioglu (2007) & $\begin{array}{l}\text { Turkey short-run elasticity of electricity demand 1968- } \\
2005\end{array}$ & -0.33 to -0.46 \\
\hline Iwayemi et al. (2010) & $\begin{array}{l}\text { Nigeria short-run elasticity of gasoline demand 1976- } \\
2006\end{array}$ & -0.25 \\
\hline Jamil et al. (2011) & $\begin{array}{l}\text { Pakistan short-run elasticity of total electricity demand } \\
2000 \mathrm{~s}\end{array}$ & -0.07 \\
\hline Lin et al. (2013) & $\begin{array}{l}\text { China medium-run elasticity of gasoline demand 1997- } \\
2008\end{array}$ & -0.196 to -0.497 \\
\hline Nahata et al. (2007) & $\begin{array}{l}\text { Russia short-run elasticity of electricity demand } 1995 \text { - } \\
2000\end{array}$ & -0.165 to -0.28 \\
\hline Ramanathan (1999) & India short-run elasticity of gasoline demand 1972-1993 & -0.21 \\
\hline Sene (2012) & $\begin{array}{l}\text { Senegal short-run elasticity of gasoline demand 1970- } \\
2008\end{array}$ & -0.12 \\
\hline Zein-Elabdin (1997) & $\begin{array}{l}\text { Sudan short-run elasticity of charcoal demand 1960- } \\
1990\end{array}$ & -0.55 \\
\hline Ziramba (2008) & $\begin{array}{l}\text { South Africa short-run elasticity of electricity demand } \\
1978-2005\end{array}$ & -0.02 \\
\hline
\end{tabular}

Notes: Gulf Cooperation Council countries are Saudi Arabia, Kuwait, Bahrain, Qatar, UAE, Oman). All electricity demand elasticity estimates are for residential customers unless otherwise noted.

${ }^{\ddagger}$ We report the IV fixed effects estimate.

Finally, Gillingham (2013) examines the direct rebound effect of a policy-induced change in vehicle prices that leads to consumers purchasing different vehicles (each with bundles of attributes) and then driving them more. The result is an elasticity of driving with respect to operating costs of -0.15 for new vehicles in California. We believe that further research on the 
rebound effect of PII is very important for policy development and we hope to see more of it in the future.

\section{Effects on Other Goods}

In addition to the direct rebound, there are also substitution and income effects for other goods. Most studies aim only to estimate the income effects for other goods (calling this the indirect rebound) and to answer the question: "If consumers are given an extra dollar, how will they spend it?" 0 One approach is to assume that consumers make purchases associated with the average energy intensity of all consumer goods, an assumption often called 'proportional respending.' Studies that use this approach examine the energy intensity of the economy either using input-output tables or other aggregate statistics of economic activity and energy use. A second approach is to compare consumption patterns across income brackets using crosssectional data (Thiesen et al. 2008). A third approach is to use income elasticities that are based on how consumers change demand for goods over time as income rises (Druckman et al. 2011). The findings in the literature vary, but most recent work tends to find an estimate on the order of 5 to 15 percent (Druckman et al. 2011, Thomas et al. 2013). Thomas et al. (2013) also make assumptions to attempt to bound the substitution effects for other goods in their estimate. One would expect that these effects would vary depending on the cross-elasticities between the good in question and other energy using goods, the additional cost of the more efficient good, and any additional energy use from the production of the more efficient good. Notably, all existing estimates assume a ZCB scenario, but additional costs would change the income effects for other goods, reducing it if the product is more energy intensive than the marginal consumption bundle Moreover, most of these estimates are from developed countries, although there is some notable work on the income elasticity of energy use in developing countries (Wolfram et al. 2012).

\section{Channels of the Rebound Effect: Macroeconomic}

The macroeconomic rebound effect is complex. Markets re-equilibrate when the demand for an energy resource changes, and an increase in energy efficiency may affect overall energy demand through multiple channels of adjustment. In this section we seek to bring clarity to the topic in three ways: 1) We define the "macroeconomic rebound" and review the theoretical

\footnotetext{
${ }^{6}$ Technically, we would want to know how consumers would spend the dollar on all goods except the more energyefficient one.
} 
pathways that are thought to generate it; 2) we describe the challenges inherent in quantifying the magnitude of the macroeconomic rebound, including a discussion of the pitfalls into which common approaches stumble; and 3) we discuss our view of what is known and unknown, and what this means for environmental economics research and policymaking.

\section{Defining Macroeconomic Rebound Effects}

As defined in the literature, macroeconomic rebound effects increase energy use after an energy efficiency improvement through market adjustments and innovation channels. These are easiest if considered in the context of a ZCB, and that is what underpins much of the discussion below. However, it is theoretically possible to consider macroeconomic rebound effects in the context of a PII, although we have never seen this done. Our exposition divides macroeconomic rebound effects into a price effect and a growth effect.

\section{Macroeconomic Price Effect}

The 'macroeconomic price effect' is an economy-wide analog to the direct rebound effect that works through prices (Gillingham et al. 2013). When an energy efficiency improvement shifts the market demand curve for energy in, consumers and producers will adjust until a new equilibrium is reached. Consider the oil market. An efficiency improvement in, say, the United States will lower the global price, increasing the quantity of oil demanded on the global market. This effect can be seen graphically in Figure 1. The initial increase in energy efficiency shifts the global demand curve inward, from D to D'. Since $a$ minus $b$ is the shift in demand and $a$ minus $c$ is the change in equilibrium quantity, the macroeconomic price effect is $1-(a-c) /(a-b)$. The magnitude of this rebound effect is thus a function of the slopes of the demand and supply curves, whereby increasingly inelastic supply and increasingly elastic demand induce a higher rebound.

\section{Macroeconomic Growth Effect}

The 'macroeconomic growth effect' — an oft-cited but poorly articulated concept - is the rationale behind many of the backfire claims. ${ }^{7}$ In fact, the classic example given by Jevons

\footnotetext{
${ }^{7}$ Consider the following quote on page 8 in Jenkins et al. (2011): "The more efficient production and use of energy at a macroeconomic scale drives economic productivity overall and encourages the substitution of energy for other factors of production (e.g., labor), resulting in more rapid economic growth and energy consumption ('macroeconomic rebound' effects)."
} 
(1865) postulates a type of macroeconomic growth effect. The basic logic is that an increase in efficiency of energy-consuming durables could spur economic growth - either through a reallocation of growth through sectoral reallocation or overall growth through an increase in total factor productivity. Economic growth requires additional energy consumption. There are three main pathways by which the macroeconomic growth effect could occur.

First, sectoral reallocation due to a change in energy efficiency may occur with a change in the relative return of sectors in the economy. ${ }^{8}$ A change in the productivity of energy inputs in an energy-intensive sector may improve the relative return on investment in that sector, leading that sector to grow relative to others. This can be (roughly) thought of as the supply-side analogy to the substitution effects discussed earlier.

\section{Figure 1. Macroeconomic Price Effect}

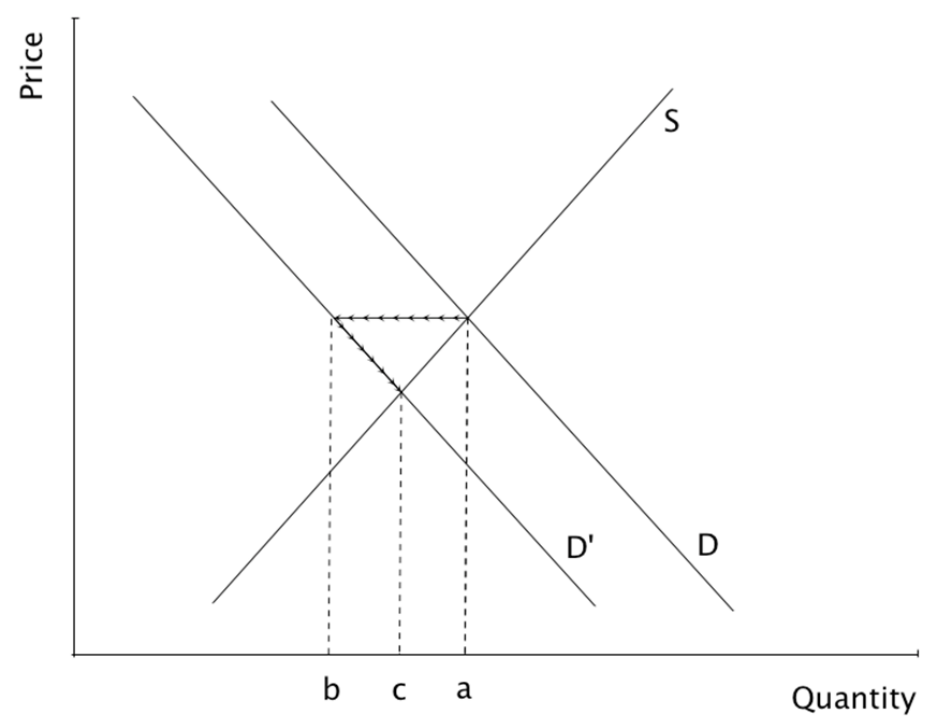

Note: This graph depicts the macroeconomic price effect associated with an energy efficiency policy that shifts demand inwards from $D$ to $D$ '. The shift in demand reduces the quantity of energy demanded from $a$ to $b$; however the equilibrium outcome yields a smaller energy reduction due to the price effect, which moves quantity from $b$ to $c$.

\footnotetext{
${ }^{8}$ Sectoral reallocation in response to changing costs is equivalent to a reallocation of inputs into aggregate production in response to changing costs.
} 
A second potential channel is induced innovation: a shock to total factor productivity. One possibility is that an energy efficiency policy (PII) leads manufacturers to update their processes, thus inducing innovation. Alternately, a ZCB in one sector may spill over to others. For example, the development of lighter-weight aircraft to improve aircraft efficiency may spill over to other sectors and lead to lighter-weight vehicles. Of course, to be accounted as a rebound, the innovation in other sectors must be attributable directly to the spillovers from the energy efficiency improvement. Should such spillovers exist, they could increase or decrease energy use in the other sectors. Whatever innovation effects occur, there is also the possibility that they are magnified (or interact in some way) with the sectoral reallocation in a manner that generates some path dependence.

A final potential channel of the macroeconomic growth effect relates to the deployment of inframarginal resources freed by a ZCB. Macroeconomists often discuss the multiplier effect of fiscal spending in a Keynesian context. One could postulate a similar channel for the aggregated indirect rebound effects: dollars that are re-spent can engage "new" economic activity that utilizes previously idle resources, causing the overall economic impact to exceed the initial amount by some multiplier (Borenstein 2015). For such a multiplier effect to occur, there must be idle resources available in the economy (such that the incremental resources do not simply crowd out private investment). This may be the case during recessions, but is less likely to be the case during economic upswings. Overall, there is severe disagreement between macroeconomists about the size of the fiscal multiplier (Ramey 2011), and in fact the multiplier in the rebound setting is slightly different, since there is long-term debt associated with fiscal stimulus, but not with a ZCB. We are not aware of any study focusing directly on estimating such multipliers in the context of energy efficiency.

\section{Quantifying Macroeconomic Rebound Effects}

Quantifying the magnitude of the macroeconomic price effect is not simple, though guidance exists in the form of estimates of demand and supply elasticities. In contrast, quantifying the macroeconomic growth effect can be quite problematic. The interconnectedness of the global economy makes it extremely difficult to deploy more reliable empirical methods, so we are often left with estimates of correlation rather than causation. Without carefully identifying the mechanisms underlying the estimation of the effect, it is all too tempting to misattribute increased energy use to an increase in energy efficiency, when in reality many other factors are at work. 


\section{Quantifying the Macroeconomic Price Effect}

The magnitude of the macroeconomic price effect depends on the relative supply and demand elasticities, as should be clear from Figure 1. If the demand elasticity is low and the supply elasticity is high, then the effect will be small. The estimates given above for the price elasticity of gasoline use suggest a relatively inelastic oil demand function, at least in the medium-run. The supply of oil is considered relatively inelastic in the short-run due to capacity constraints, but would be expected to be more elastic in the long-run, for it depends on how development of new extraction technologies responds to price. Unfortunately, there is very little empirical evidence on such supply elasticities. Borenstein (2015) uses oil supply elasticities of $0.2,0.6$, and 1.0 for a sensitivity analysis of the macroeconomic price effect using constantelasticity supply and demand curves. Given the remarkable innovations in oil extraction over the past several decades with high oil prices, we agree with Borenstein that the long-run oil supply elasticity may be rather high.

The estimates in Borenstein (2015) indicate that with an oil demand elasticity of -0.4 and an oil supply elasticity of 1.0, the macroeconomic price effect is on the order of 30 percent. We find a similar result with analogous calculations using linear demand and supply functions. The possible range is quite large: with a supply elasticity of only 0.2 and demand elasticity of -0.6 , we can expect to see a macroeconomic price effect as large as 76 percent. While we do not believe an effect this large is plausible, we acknowledge that this effect may be important in the oil market. Given the likely high long-run oil supply elasticity and low or moderate demand elasticity, we suspect a macroeconomic price rebound on the order of 20 to 30 percent in oil markets. However, we have not yet seen evidence on other energy markets, such as electricity or natural gas. For all markets, it is important to recognize that the macroeconomic price effect will always be less than one. As long as we have a downward sloping demand curve and upward sloping supply curve, backfire due solely to the macroeconomic price effect is theoretically impossible.

\section{Quantifying the Macroeconomic Growth Effect}

Despite its centrality to backfire claims, the macroeconomic growth effect is the area of research with the least amount of concrete evidence. Attempts to quantify the macroeconomic growth effect are plagued by the same challenges that are encountered in most macroeconomics research. Our global economy is a single, interconnected, complex dynamic system, rendering economists' most reliable micro-empirical techniques ineffective. It is thus nearly impossible to make dispositive arguments about cause and effect in this setting. That is, we cannot say with 
empirical certainty how US fuel economy standards affect long-run energy use in the United States, let alone in China.

Fortunately, basic economic theory provides some profound guidance on the macroeconomic growth rebound most commonly discussed: sectoral reallocation. The key insight is that the extent to which a ZCB leads to increases or decreases in overall energy use depends on the elasticities of substitution in consumption and production. To see this, consider the following simple example. A household consumes two goods, an aggregate consumption good (e.g., food or clothing) and an energy service (e.g., driving). So households can use their income to either purchase the consumption good or to purchase a car and energy to power the car. The ZCB question is as follows: "what happens to aggregate energy use in the economy if cars are made more energy efficient?"

In the consumer sector, the answer depends on the elasticity of substitution between goods and energy services in the household utility function. Consider the extremes. If goods and energy services are perfect substitutes, then the household will spend its entire budget on whichever good has the highest utility per dollar spent. If energy services become less expensive than goods (in utils per dollar), then the household may shift its entire budget in that direction. On the other hand, if goods and energy services are perfect complements, then they will optimally be consumed in fixed proportion. In that case, making one of the goods marginally cheaper (e.g. through energy efficiency standards) will make little difference in consumption and overall energy use. Importantly, since energy is a derived demand from energy services, ZCB may cause the level of energy services to increase, but still require less energy than initially needed. In fact, there must be a high degree of substitution towards energy services in consumption for the level of actual energy use to increase above pre-energy-efficiency levels.

So far, this logic is the same as the logic behind the microeconomic substitution effects, and for that reason the consumer substitution effects will be subsumed in estimates of the sectoral reallocation effect. But sectoral reallocation is even broader; it depends not only on patterns in consumption, but also in production. For production, precisely the same logic applies. Where production occurs by combining energy inputs with non-energy inputs (e.g. capital and labor), the degree of substitutability/complementarity in production determines the overall effect of ZCB on energy use. If the inputs are highly substitutable, an increase in energy efficiency in production will cause a large swing towards increasing energy inputs. If they are complements, they must be used in fixed proportion, and energy demand will remain unchanged. 
A nice implication of these insights is that the sectoral reallocation rebound is largely driven by the magnitude of substitution elasticities. Our intuition leads us to view energy and non-energy inputs as more complementary than substitutable in both consumption and production. Goulder et al. (1999) share this intuition. Its simulation model of alternate abatement policies assumes complementarity of energy and other inputs to production. ${ }^{9}$ This leads us to believe that macroeconomic growth rebound effects may be small. However, there is clearly room in the literature for more research to better quantify the relevant substitution elasticities.

\section{Literature on the Macroeconomic Growth Effect}

The above insights are particularly useful to keep in mind when interpreting the literature on the macroeconomic growth effect, which focuses primarily on sectoral reallocation. Other channels may be implicitly subsumed in the macroeconomic growth effect, but to the best of our knowledge have not been identified separately. There are three classes of papers in the literature quantifying the macroeconomic growth rebound.

The first class of papers involves a structural model of the production function of the economy that is used to make theoretical predictions about the rebound effect. The second attempts to econometrically estimate the total rebound effect (macroeconomic and microeconomic) using historical time series data. The third involves simulation models of the economy based on input-output tables of economic activity and calibrated relationships between key variables governing economic growth.

Beginning with Saunders (1992), there has been stream of papers in the energy economics literature relying on the Solow growth model to provide theoretical insight into the sectoral reallocation rebound. For example, Saunders (1992) examines how energy efficiency improvements affect overall energy consumption using a single sector Solow growth model that includes capital, labor and energy inputs. In this simple setting, the consumer considers energyintensive goods as perfect substitutes for non-energy-intensive goods. Thus, by construction, Saunders finds that backfire can occur.

Our concern with this model, and many others in this literature, is that it relies heavily on structural assumptions. For example, switching to a Leontief production function (perfect

\footnotetext{
${ }^{9}$ Goulder et al. (1999) assumes an elasticity of substitution of 0.8 . It may be even lower in our context, since the energy efficiency intervention itself will already dictate substitution towards more energy efficient production technology.
} 
complementarity of inputs) would immediately imply zero rebound. Of course, this is an equally restrictive structural assumption as a single sector Solow growth model. While such theoretical exercises are interesting, nearly any outcome is possible by carefully choosing structural assumptions and functional forms.

This observation should not be surprising to macroeconomists, but it means that numerical investigations are all the more important to provide real guidance on the magnitude of all three of the channels of the macroeconomic growth rebound. Yet this is where causal attribution is critical-and extremely difficult. For the last century, we have seen large increases in energy use and in the energy efficiency of a variety of durable goods. To claim a causal relationship between energy efficiency and energy use requires demonstrating that energy consumption has not increased due to some other factor. Ideally, the experiment needed to identify a ZCB would have a world with the ZCB and a world without. Unfortunately, just as for many questions in macroeconomics, such an experiment is impossible. In fact, it is extremely difficult, if not impossible, to disentangle the effect of energy efficiency improvements from exogenous economic growth and dramatic improvements in energy services that occurred at the same time. Not surprisingly, the few econometric investigations using historical data to find evidence of a combined rebound effect leading to backfire (e.g., Tsao et al. (2010) and Saunders (2013)) are not in economics journals, where the standard for empirically identifying a causal effect tends to be higher.

In the absence of credible identification, macroeconomists often build models of the economy to simulate the effects of policies. This brings us to the third class of approaches used to estimate the macroeconomic rebound effect: calibrated simulation models. These models tend to be general equilibrium models based on input-output tables of economic activity or estimated macro-econometric models with hundreds of equations. Of course, the results of such models are driven by the structure of the model and the parameterization of the relationships. For this reason, many macroeconomic modelers focus on modeling for intuition, rather than numerical estimates.

The simulation models that are used to numerically estimate the macroeconomic rebound effect perturb energy efficiency and compare the total energy consumption in the scenario to the energy consumption in the business-as-usual case. If the change in predicted energy use is less than the expected effect of energy efficiency, the difference is attributed to rebound; if total energy increases, it is consistent with backfire. Some of the most interesting papers in this literature build computable general equilibrium or econometric simulation models of the U.K. economy (Barker et al. 2009, Barker et al. 2007, Turner 2009), and find results ranging from 
negative rebounds to massive backfire. The vast range of results may be useful for highlighting what different combinations of structural assumptions and parameter values imply for the macroeconomic rebound effect. But their reliance on correlations as structural parameters leave us unconvinced that they provide real guidance as to the magnitude of the effect. These issues are challenging, and clearly a valuable area for future research that combines clever empirical approaches with carefully thought-out numerical simulations.

\section{Discussion}

In the face of these challenges, how should we view the magnitude of the macroeconomic rebound effect? Recall first that estimates of the sectoral reallocation macroeconomic rebound are not necessarily additive with respect to the microeconomic rebound effects, which are typically already aggregated into the macroeconomic measure. Moreover, the macroeconomic price and sectoral reallocation effects and may even be partly offsetting, for sufficiently lower equilibrium energy prices may lead to a reallocation away from energy (Turner 2009). Furthermore, to the extent that the numerical simulations are based on historical correlations, rather than causal effects, we should be cautious of interpreting the exact point estimates too literally.

That said, the macroeconomic growth effect may be substantial in certain circumstances, and it is likely that there is at least some increase in energy consumption from the macroeconomic growth effect. Thus, at this point, when considering a ZCB, perhaps the best approach for a policy economist is to calculate the macroeconomic price effect based on the best estimates of elasticities, and then perform a sensitivity analysis with different values of the macroeconomic growth rebound effect. Two recent estimates of the macroeconomic growth rebound that one could consider using for such a sensitivity analysis are 11 percent (Barker et al. 2007) or 21 percent (Barker et al. 2009). ${ }^{10}$ In our view, the literature does not provide convincing evidence for backfire due to the macroeconomic rebound effect.

What does a macroeconomic rebound imply about the welfare effects of policy? The price effect comes about from reaching equilibria in markets, which improves welfare. Sectoral reallocation leads to more efficient production in an economy, improving welfare. If the energy efficiency improvement induces innovation, this also would improve welfare. These welfare

\footnotetext{
10 This 21 percent is based on the 2020 estimate, while the 2030 estimate is 41 percent. However both include the income effect within the macroeconomic rebound.
} 
gains may be countered by losses from greater external costs of production or consumption, so the net welfare effects are ambiguous.

\section{Conclusions and Lessons for Policy}

The magnitude of the rebound effect is a debate that persists, with important implications for energy efficiency policy. Yet the rebound effect has many facets, and the increasingly voluminous literature has become confusing and difficult to translate into policy relevance. This paper attempts to make three basic contributions. First, we introduce the important conceptual distinction between a rebound effect associated with a costless energy efficiency improvement that holds other attributes constant (ZCB), and an energy efficiency policy that may be bundled with other product changes that affect energy use (PII). Second, we distill the empirical literature on the microeconomic rebound into a manageable number of estimates that we view as the most reliable. Third, we attempt to clarify the nature of the macroeconomic rebound, and discuss how one might go about conceptualizing (or estimating) the size of the effect.

The existing literature does not provide support for claims that energy efficiency gains will be reversed by the rebound effect. The total microeconomic rebound is, in most cases, on the order of 20 to 40 percent when including all substitution and income effects and perhaps even including the embodied energy in the energy efficiency improvement. Far less is known (or knowable) about the macroeconomic rebound. We articulate a framework for thinking about these effects that lead to three observations. First, in some markets the macroeconomic price effect may be substantial, but must be less than 100 percent. Second, rebound based on sectoral reallocation is likely smaller, since energy is more likely to be a complement to, rather than substitute for, other inputs in production. Finally, little is known about the effects of induced innovation and productivity on rebound. The literature exploring how regulation affects total factor productivity lacks consensus. Regardless, if induced innovation and productivity lead to rebound, quantifying the effect engenders the difficult task of determining a counterfactual path of innovation and productivity. There is currently scant evidence to support this induced innovation channel, but we see this as another area for future research.

The cumulative effect of these channels of rebound in a ZCB setting may be large in some situations and smaller in others, even if the evidence does not support backfire. If pressed to offer our subjective assessment, in most cases we do not expect the total rebound effect to 
exceed 60 percent, but we recognize that it is possible to have a larger total effect. ${ }^{11}$ One might expect a PII to have a larger rebound due to associated changes in product attributes that consumers value, but a smaller rebound to the extent that the cost of the policy mitigates both the income and macroeconomic growth effects. In fact, sufficiently costly energy efficiency policies may well engender negative rebound effects.

This underscores our primary conclusion: rather than focusing on the rebound and backfire, it is more useful to focus on the economic efficiency of energy efficiency policies in the broadest possible sense. The rebound effect is only one component that factors into the equation. More importantly, it is also a factor that in most cases leads to welfare gains. This is especially true for the one rebound aspect that is hardest to measure: induced innovation and productivity growth from an energy efficiency policy. Should it indeed occur as a direct result of a particular energy efficiency policy, it would only enhance welfare. More broadly, unless there are severe external costs from the rebound, rebound would be a benefit, not a cost.

Rather than consider the rebound effect as a deterrent from passing energy efficiency policies, policymakers should include these welfare gains in the tally of benefits of a policy. The mistake of designing policies to 'mitigate' the rebound effect stems from a focus on minimizing energy use, rather than the broader objective of maximizing economic efficiency. In sum, while the energy savings from energy efficiency policies will be reduced by the presence of a rebound effect, a ZCB rebound is likely to both conserve energy and increase welfare. The same may be true for a PII rebound, but each policy will require its own analysis.

\footnotetext{
11 This 60 percent estimate is based on a 30 percent long-run microeconomic rebound, 25 percent macroeconomic price effect, and 5 percent macroeconomic growth effect (accounting for the fact that estimates of the macroeconomic growth effect both range widely and may be implicitly including some of the other rebounds), but the breakdown may vary by context.
} 


\section{References}

Al-Faris, Abdul Razak. 2002. "The Demand for Electricity in the GCC Countries." Energy Policy 30 (2): 117-124.

Allcott, Hunt. 2011. "Rethinking Real-Time Electricity Pricing." Resource and Energy Economics 33 (4): 820-842.

Alves, Denisard, and Rodrigo De Losso da Silveira Bueno. 2003. "Short-run, long-run and Cross Elasticities of Gasoline Demand in Brazil." Energy Economics 25 (2): 191-199.

Atakhanova, Zauresh, and Peter Howie. 2007. "Electricity Demand in Kazakhstan." Energy Policy 35 (7): 3729-3743.

Athukorala, Wasantha, and Clevo Wilson. 2010. "Estimating Short and Long-term Residential Demand for Electricity: New Evidence from Sri Lanka." Energy Economics 32 (S1): S34-S40.

Azevedo, Ines L. 2014. "Consumer End-Use Energy Efficiency and Rebound Effects." Annual Review of Environment and Resources forthcoming.

Barker, Terry, Athanasios Dagoumas, and Jonathan Rubin. 2009. "The Macroeconomic Rebound Effect and the World Economy." Energy Efficiency 2 (4): 411-427.

Barker, Terry, Paul Ekins, and Timothy Foxon. 2007. "The macro-economic rebound effect and the UK economy." Energy Policy 35 (10): 4935-4946.

Barla, Philippe , Bernard Lamonde, Luis F. Miranda-Moreno, and Nathalie Boucher. 2009. "Traveled Distance, Stock and Fuel Efficiency of Private Vehicles in Canada: Price Elasticities and Rebound Effect." Transportation 36 (4): 389-402.

Ben Sita, Bernard, Walid Marrouch, and Salah Abosedra. 2012. "Short-run Price and Income Elasticity of Gasoline Demand: Evidence from Lebanon." Energy Policy 46: 109-115.

Borenstein, Severin. 2015. "A Microeconomic Framework for Evaluating Energy Efficiency Rebound and Some Implications." Energy Journal forthcoming.

Chan, Nathan, and Kenneth Gillingham. 2014. "The Microeconomic Theory of the Rebound Effect and its Welfare Implications." Journal of the Association of Environmental and Resource Economists. 
Crotte, Amado, Robert Noland, and Daniel Graham. 2010. "An Analysis of Gasoline Demand Elasticities at the National and Local Levels in Mexico." Energy Policy 38 (8): 44454456.

Davis, Lucas. 2008. "Durable Goods and Residential Demand for Energy and Water: Evidence from a Field Trial." RAND Journal of Economics 39 (2): 530-546.

Davis, Lucas, Alan Fuchs, and Paul Gertler. 2015. "Cash for Coolers: Evaluating a Large-Scale Appliance Replacement Program in Mexico." American Economic Journal: Economic Policy forthcoming.

Druckman, Angela, Mona Chitnis, Steve Sorrell, and Tim Jackson. 2011. "Missing Carbon Reductions? Exploring Rebound and Backfire Effects in UK Households." Energy Policy 39 (6): 3575-3581

Frondel, Manuel, and Colin Vance. 2013. "Re-Identifying the Rebound: What about Asymmetry?" Energy Journal 34 (4): 43-54.

Gillingham, Kenneth. 2011. The Consumer Response to Gasoline Price Changes: Empirical Evidence and Policy Implications, Stanford University Ph.D. Dissertation.

Gillingham, Kenneth. 2013. "Selection on Anticipated Driving and the Consumer Response to Changing Gasoline Prices." Yale University Working Paper.

Gillingham, Kenneth. 2014. "Identifying the Elasticity of Driving: Evidence from a Gasoline Price Shock." Regional Science \& Urban Economics 47 (4): 13-24.

Gillingham, Kenneth, Matthew Kotchen, David Rapson, and Gernot Wagner. 2013. "The Rebound Effect is Over-played." Nature 493: 475-476.

Gloger, Stefan. 2011. "Policies to Overcome the Rebound Effect - A New Challenge for Environmental Policy." Technical Report, Ministry of the Environment, BadenWuerttemberg.

Goulder, Lawrence, Ian Parry, Roberton Williams, and Dallas Burtraw. 1999. "The Costeffectiveness of Alternative Instruments for Environmental Protection in a Second-best Setting." Journal of Public Economics 72 (3): 329-360.

Greening, Lorna A., David L. Greene, and Carmen Difiglio. 2000. "Energy efficiency and consumption — the rebound effect—a survey." Energy Policy 28 (6-7): 389-401.

Halicioglu, Ferda. 2007. "Residential Electricity Demand Dynamics in Turkey." Energy Economics 29 (2): 199-210. 
Hamilton, James. 2009. "Understanding Crude Oil Prices." Energy Journal 30 (2): 179-206. Hughes, Jonathan, Christopher Knittel, and Daniel Sperling. 2008. "Evidence of a Shift in the Short-Run Price Elasticity of Gasoline Demand." Energy Journal 29 (1): 93-114.

Ito, Koichiro. 2014. "Do Consumers Respond to Marginal or Average Price? Evidence from Nonlinear Electricity Pricing." American Economic Review forthcoming.

Iwayemi, Akin, Adeola Adenikinju, and Adetunji Babatunde. 2010. "Estimating Petroleum Products Demand Elasticities in Nigeria: A Multivariate Cointegration Approach." Energy Economics 32 (1): 73-85.

Jamil, Faisal, and Eatzaz Ahmad. 2011. "Income and Price Elasticities of Electricity Demand: Aggregate and Sector-wise Analyses." Energy Policy 39 (9): 5519-5527.

Jenkins, Jesse, Ted Nordhaus, and Michael Shellenberger. 2011. Energy emergence: rebound and backfire as emergent phenomena. Oakland, CA: Breakthorugh Institute.

Jessoe, Katrina, and David Rapson. 2013. "Knowledge is (Less) Power: Experimental Evidence from Residential Energy Use." American Economic Review 104 (4): 1417-1438.

Jevons, William Stanley. 1865. The coal question; an enquiry concerning the progress of the Nation, and the probable exhaustion of our coal-mines. $2 \mathrm{~d}$ ed. London,: Macmillan.

Li, Shanjun, Joshua Linn, and Erich Muehlegger. 2014. "Gasoline Taxes and Consumer Behavior." American Economic Journal: Economic Policy forthcoming.

Lin, C.-Y. Cynthia, and Jieyin Zeng. 2013. "The Elasticity of Demand for Gasoline in China." Energy Policy 59 (C): 189-197.

Linn, Joshua. 2013. "The Rebound Effect for Passenger Vehicles." RFF Working Paper.

Nahata, Babu, Alexei Izyumov, Vladimir Busygin, and Anna Mishura. 2007. "Application of the Ramsey Model in Transition Economy: A Russian Case Study." Energy Economics 29 (1): 105-125.

Ramanathan, Raj. 1999. "Short- and Long-run Elasticities of Gasoline Demand in India: An Empirical Analysis Using Co-Integration Techniques." Energy Economics 21 (4): 321330.

Ramey, Valerie. 2011. "Can Government Purchases Stimulate the Economy." Journal of Economic Literature 49 (3): 673-685. 
Saunders, Harry. 1992. "The Khazzoom-Brookes Postulate and Neoclassical Growth." Energy Journal 13 (4): 131-148.

Saunders, Harry. 2013. "Historical Evidence for Energy Consumption Rebound in 30 US Sectors and a Toolkit for Rebound Analysts." Technological Forecasting and Social Change 80 (7): 1317-1330.

Sene, Seydina Ousmane. 2012. "Estimating the Demand for Gasoline in Developing Countries: Senegal." Energy Economics 34 (1): 189-194.

Small, Kenneth A., and Kurt van Dender. 2007. "Fuel Efficiency and Motor Vehicle Travel: The Declining Rebound Effect." Energy Journal 28 (1): 25-51.

Sorrell, Steve. 2007. "The Rebound Effect: An Assessment of the Evidence for Economy-wide Energy Savings from Improved Energy Efficiency." UK Energy Research Center Report.

Sorrell, Steve , and John Dimitropoulos. 2008. "The rebound effect: Microeconomic definitions, limitations and extensions." Ecological Economics 65 (3): 636-649.

Thiesen, J., T. Christensen, T. Kristensen, R. Andersen, B. Brunoe, T. Gregersen, M. Thrane, and B. Weidema. 2008. "Rebound Effects of Price Differences." International Journal of Life Cycle Assessment 13 (2): 104-114.

Thomas, Brinda A., and Ines L. Azevedo. 2013. "Estimating Direct and Indirect Rebound Effects for US Households With Input-Output Analysis. Part 2: Simulation." Ecological Economics 86 (C): 188-198.

Tsao, Jeffrey, Harry Saunders, J. Randall Creighton, Michael Coltrin, and Jerry Simmons. 2010. "Solid-state Lighting: An Energy-economics Perspective." Journal of Physics D: Applied Physics 43: 1-17.

Turner, Karen. 2009. "Negative Rebound and Disinvestment Effects in Response to an Improvement in the UK Economy." Energy Economics 31 (5): 648-666.

van den Bergh, Jeroen. 2011. "Energy Conservation More Effective with Rebound Policy." Environmental and Resource Economics 48 (1): 43-58.

West, Sarah. 2004. "Distributional Effects of Alternative Vehicle Pollution Control Technologies." Journal of Public Economics 88 (3-4): 735-757.

Wolfram, Catherine, Orie Shelef, and Paul Gertler. 2012. "How Will Energy Demand Develop in the Developing World?" Journal of Economic Perspectives 26 (1): 119-138. 
Zein-Elabdin, Eiman. 1997. "Improved Stoves in Sub-Saharan Africa: The Case of the Sudan." Energy Economics 19 (4): 465-475.

Ziramba, Emmanuel. 2008. "The Demand for Residential Electricity in South Africa." Energy Policy 36 (9): 3460-3466. 